\title{
Wireless Transmission of Electrical Power Overview of Recent Research \& Development
}

\author{
Sagolsem Kripachariya Singh, T. S. Hasarmani, and R. M. Holmukhe
}

\begin{abstract}
The aim of this research work is to give a overview of recent researches and development in the field of wireless power transmission. The methods applied for wireless power transmission like Induction, Electromagnetic transmission, Evanescent wave coupling, Electrodynamic induction, Radio and microwave and Electrostatic Induction ,are discussed.This study also focuses on the latest technologies, merits and demerits in this field. The economic aspects are briefly discussed.
\end{abstract}

Index Terms-wireless transmission, witricity, power, solarpower

\section{INTRODUCTION}

In the past, product designers and engineers have faced challenges involving power: the continuity of supplied power, recharging batteries, optimizing the location of sensors, and dealing with rotating or moving joints. Although those challenges remain, new demands that arise from increased use of mobile devices and operation in dirty or wet environments mean that designers require new approaches to supplying power to equipment.Wireless Power Transmission from the time of Tesla has been an underdeveloped technology. Tesla had always tried to introduce worldwide wireless power distribution system. But due to lack of funding and technology of that time, he was not able to complete the task. Then onwards this technology has not been developed up to the level which would be completely applicable for practical purpose. Research has always been going on and recent developments have been observed in this field. Despite advances wireless power transmission has not been adopted for commercial use. Highlight a

\section{LITERATURE SURVEY}

- In 1864, James C. Maxwell predicted the existence of radio waves by means of mathematical model.

- In 1884, John H. Poynting realized that the Poynting Vector would play an important role in quantifying the electromagnetic energy.

- In 1888, bolstered by Maxwell's theory, Heinrich Hertz first succeeded in showing experimental evidence of radio waves by his spark-gap radio transmitter. The prediction and Evidence of the radio wave in the end of 19th century was start of the wireless power transmission.

- Nikola Tesla has been the pioneer in the field of wireless transmission of electrical power [1]. He started efforts on

Manuscript received February 25, 2011; revised March 30, 2012.

The authors are with from Bharati Vidyapeeth Deemed University College of Engineering, Pune, India. wireless transmission at 1891 in his "experimental station" at Colorado. Nikola Tesla successfully lighted a small incandescent lamp by means of a resonant circuit grounded on one end. A coil outside laboratory with the lower end connected to the ground and the upper end free. The lamp is lighted by the current induced in the three turns of wire wound around the lower end of the coil.

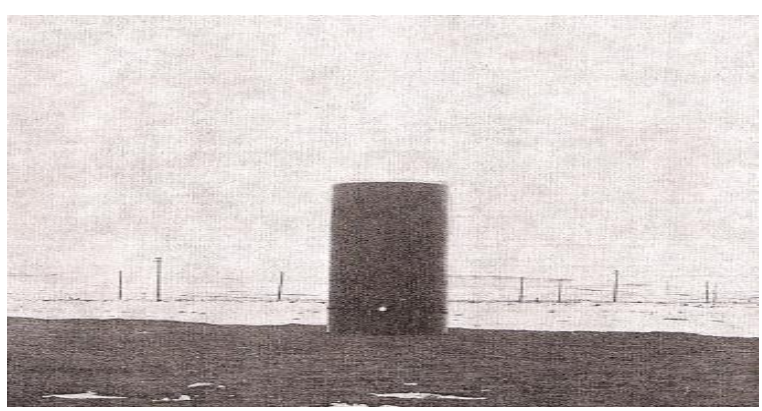

Fig. 1. Tesla's experimental lamp.

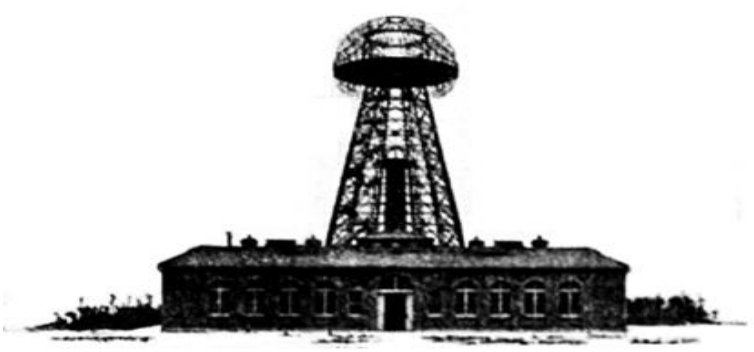

Fig. 2. Wardenclyffe tower also known as tesla's tower (187 foot) at long Island, New York.

Wardenclyffe tower was designed by Tesla for trans-Atlantic wireless telephony and also for demonstrating wireless electrical power transmission

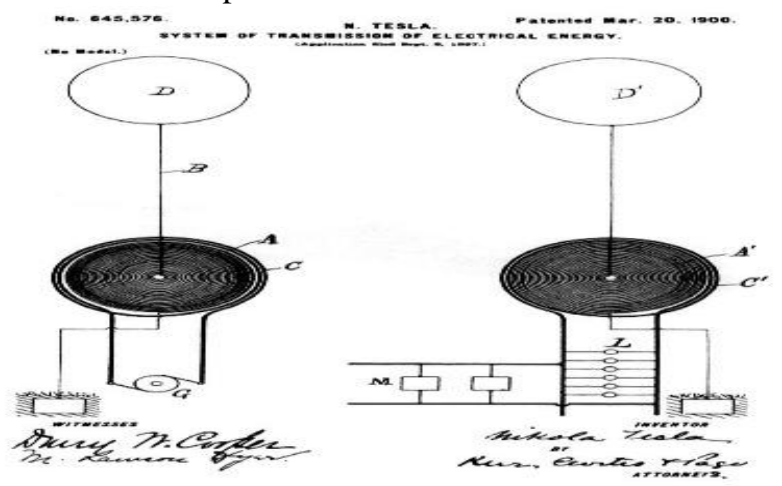

Fig. 3. Tesla's system

- William C. Brown contributed much to the modern development of microwave power transmission which dominates research and development of wireless 
transmission today. In the early 1960s brown invented the rectenna which directly converts microwaves to DC current. He demonstrated its ability in 1964 by powering a helicopter from the solely through microwaves.

- Hidetsugu Yagi a Japanese electrical engineer also tried unsuccessfully to introduce a wireless power transmission system.

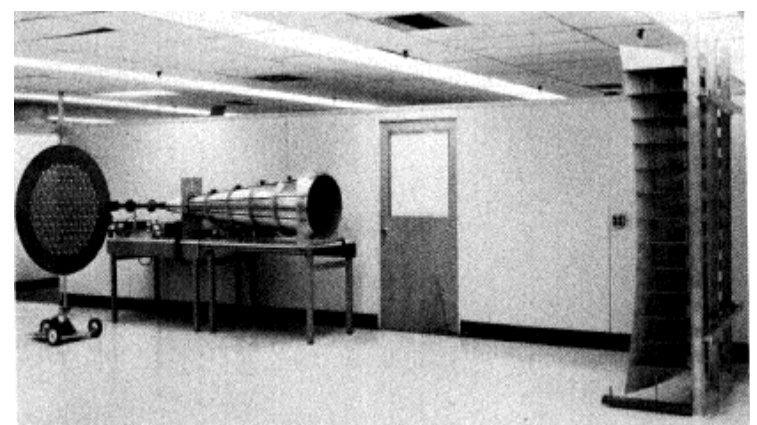

Fig. 4. Microwave power transmission laboratory experiment in 1975 by W.Brown.

\section{METHODS OF WIRELESS TRANSMISSION OF ELECTRICAL} POWER

\section{A. Induction}

The principle of mutual induction between two coils can be used for the transfer of electrical power without any physical contact in between. The simplest example of how mutual induction works is the transformer, where there is no physical contact between the primary and the secondary coils. The transfer of energy takes place due to electromagnetic coupling between the two coils. [7]

\section{B. Electromagnetic Transmission}

Electromagnetic waves can also be used to transfer power without wires. By converting electricity into light, such as a laser beam, then firing this beam at a receiving target, such as a solar cell on a small aircraft, power can be beamed to a single target. This is generally known as "power beaming".

\section{Evanescent Wave Coupling}

Researchers at MIT believe they have discovered a new way to wirelessly transfer power using non-radiative electromagnetic energy resonant tunneling. Since the electromagnetic waves would tunnel, they would not propagate through the air to be absorbed or wasted, and would not disrupt electronic devices or cause physical injury like microwave or radio transmission. Researchers anticipate up to 5 meters of range.

\section{Electrodynamic Induction}

Also known as "resonant inductive coupling" resolves the main problem associated with non-resonant inductive coupling for wireless energy transfer; specifically, the dependence of efficiency on transmission distance. When resonant coupling is used the transmitter and receiver inductors are tuned to a mutual frequency and the drive current is modified from a sinusoidal to a non-sinusoidal transient waveform. Pulse power transfer occurs over multiple cycles. In this way significant power may be transmitted over a distance of up to a few times the size of the transmitter.

\section{E. Radio and Microwave}

Power transmission via radio waves can be made more directional, allowing longer distance power beaming, with shorter wavelengths of electromagnetic radiation, typically in the microwave range. A rectenna may be used to convert the microwave energy back into electricity. Rectenna conversion efficiencies exceeding $95 \%$ have been realized. Power beaming using microwaves has been proposed for the transmission of energy from orbiting solar power satellites to Earth and the beaming of power to spacecraft leaving orbit has been considered.

\section{F. Electrostatic Induction}

Also known as "capacitive coupling" is an electric field gradient or differential capacitance between two elevated electrodes over a conducting ground plane for wireless energy transmission involving high frequency alternating current potential differences transmitted between two plates or nodes

\section{CurRent Technology IN THE FIELD OF WIRELESS POWER TRANSMISSION}

\section{A. Microwave Transmitter}

The most current research and proposals use microwaves as the frequency range of choice for transmission. At present an efficiency of $76 \%$ is possible using current technology for microwave power transmission. For transmission efficiency the waves must be focused so that all the energy transmitted by the source is incident on the wave collection device. Higher frequencies are also impractical because of the high cost of transmitters and the relative low efficiency of current optical and infrared devices.

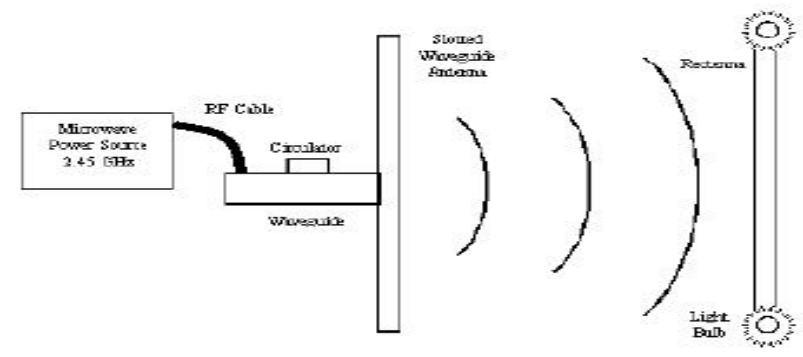

Fig. 5. Microwave transmitter

The most common transmitters for microwaves are the travelling wave tube (TWT), klystron and magnetron. The TWT is far too expensive and power restrictive making it impractical for the task of power transmission. The klystron has been the DC to microwave converter of choice however it is also somewhat expensive. Many researchers are looking to use magnetrons instead because they are cheap and efficient. Magnetron frequency output is not as precisely controllable as the klystron or TWT but power transmission is more lenient to frequency fluctuations than communication systems are. One of the more common proposals would be for an array of magnetrons to be used as the transmitter. One of the main advantages to using many smaller magnetrons as opposed to a few klystrons is that $300 \mathrm{~W}$ to $1 \mathrm{~kW}$ magnetrons are already 
mass produced for microwave ovens. The efficiency of magnetrons is inconsistently reported.

\section{B. Use of Microwave Power Transmission in Solar Power Satellites (SPS)}

Solar power generating satellites launched into space and transmitting power to Earth stations. This idea was first proposed in 1968 and all of the experiments have only been carried out in terrestrial laboratories. The SPS satellites would be put in high earth orbit at geosynchronous location. This would allow them to receive light $99 \%$ of the year. A large rectenna array facility will be built on the Earth to collect the incoming microwaves. To maintain a good lock on the rectenna the satellite will need to be built with a retrodirective transmitter which locks on to a pilot beam emanated from the ground station.

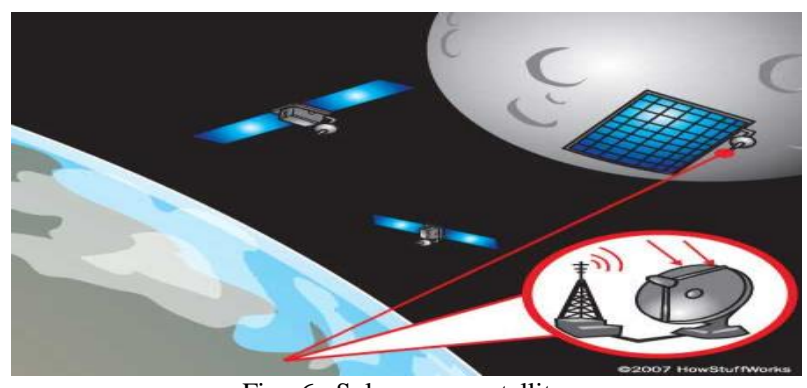

Fig. 6. Solar power satellite.

Since most of the research is done in the $2.4 \mathrm{GHz}$ to 5.8 $\mathrm{GHz}$ range there are some spectrum regulatory issues to deal with. Also since the retro directive antenna system is unproven. There is the health concern that the microwave beam could veer off target and microwave some unsuspecting family. However, a Japanese government agency is planning to send up 10 to $100 \mathrm{~kW}$ low earth orbit satellite to prove its feasibility. [2]

\section{LATEST INVENTION AND EXPERIMENTS}

\section{A. WriTricity}

The new technology called WiTricity is based on using coupled resonant objects [10]. Two resonant objects of the same resonant frequency tend to exchange energy efficiently, while interacting weakly with extraneous off-resonant objects.

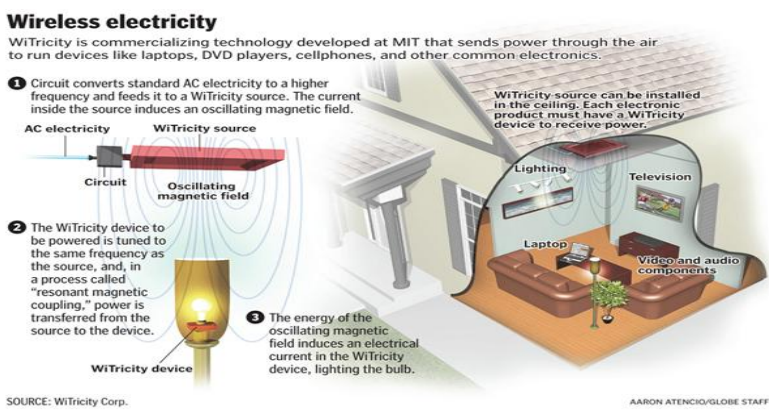

Fig. 7. WiTricity image of how the fields work.

A child on a swing is a good example of this. A swing is a type of mechanical resonance, so only when the child pumps her legs at the natural frequency of the swing is she able to impart substantial energy.

The investigated design consists of two copper coils, each a self-resonant system. One of the coils, attached to the power source, is the sending unit. Instead of irradiating the environment with electromagnetic waves, it fills the space around it with a non-radiative magnetic field oscillating at $\mathrm{MHz}$ frequencies. The non-radiative field mediates the power exchange with the other coil (the receiving unit), which is specially designed to resonate with the field. The resonant nature of the process ensures the strong interaction between the sending unit and the receiving unit, while the interaction with the rest of the environment is weak.

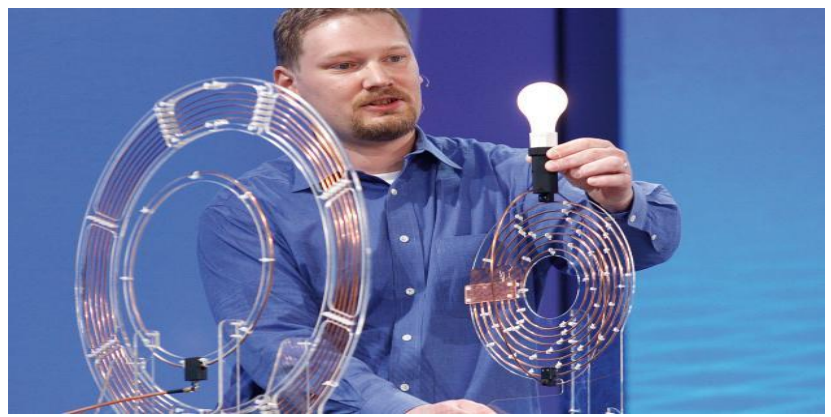

Fig. 8. .Experiment at MIT for WPT.

\section{B. Intel's Demonstration}

Intel demonstrated a captivating technology that has the potential to eliminate the need for power cords, chargers or batteries. During the presentation at yesterday's Intel Developer Forum (IDF), the chip maker wirelessly powered a 60 watt light globe from three feet away with 75 percent efficiency.The technology works by creating resonance between two magnetic fields, known as a "resonant induction" phenomenon. Intel hopes to one day use the technology to power laptops and other portable devices, either directly via a transmitter or by charging internal super capacitors which can be rapidly recharged.The research project at Intel, led by Joshua R. Smith aims to build upon this work.

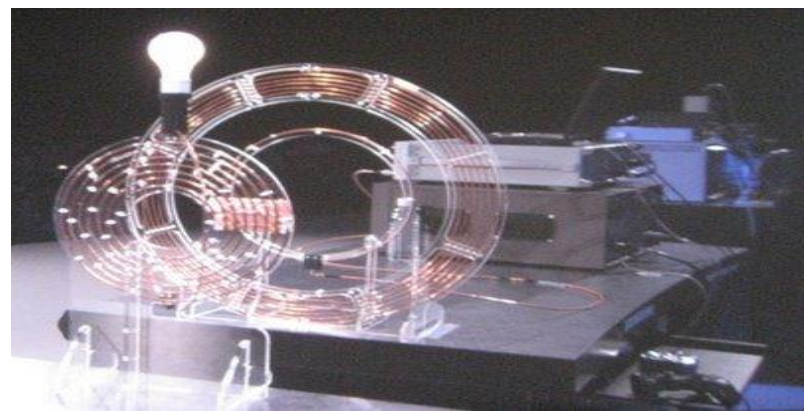

Fig. 9. Experiment at INTEL for WPT.

Lightning Lab accidentally transferred a large amount of energy while testing a high-frequency transformer.

The Navada Lightning Laboratory noticed another transformer across the lab beginning to "smoke profusely" during the test. They found the transformer was physically disconnected from its power source and set out to try and learn what had happened.

They discovered that even widely spaced coils were capable of wirelessly transmitting large amounts of power and to test it out, they rigged up a board of twenty $40 \mathrm{~W}$ light 
globes with the transmitter and receiver 5 meters $(16.4 \mathrm{ft})$ apart.

The major drawback, as is always the case with wireless power transmission, is the amount of energy lost during the process. The transmitting coil was operating at $3.6 \mathrm{KW}$ for the receiver to capture the $800 \mathrm{~W}$, which is quite inefficient.

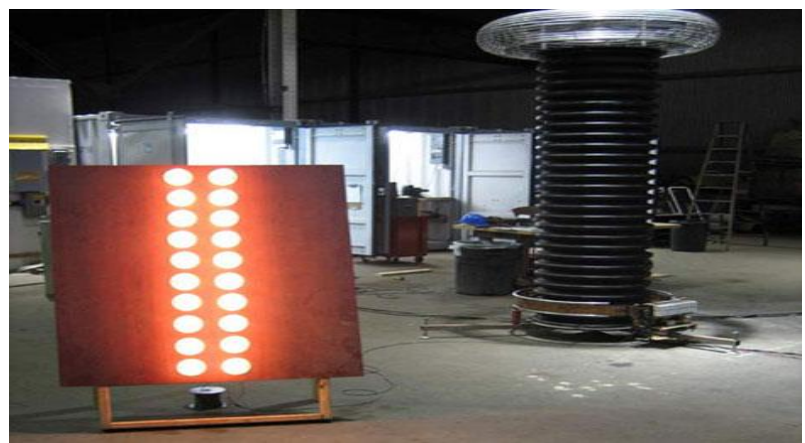

Fig. 10. Lightining lab experiment.

\section{RECENT RESEARCH}

Researches have been going on in the field of using microwave power transfer and many technologies are being developed around the globe.

1. Antennas: - In some MPT experiments in Japan, the phased array antenna was adopted to steer a direction of the microwave beam.

2. Transmitters: - Magnetron, travelling wave tube amplifiers, klystron, semiconductor amplifiers.

3. Beam control, Target detection, Propagation
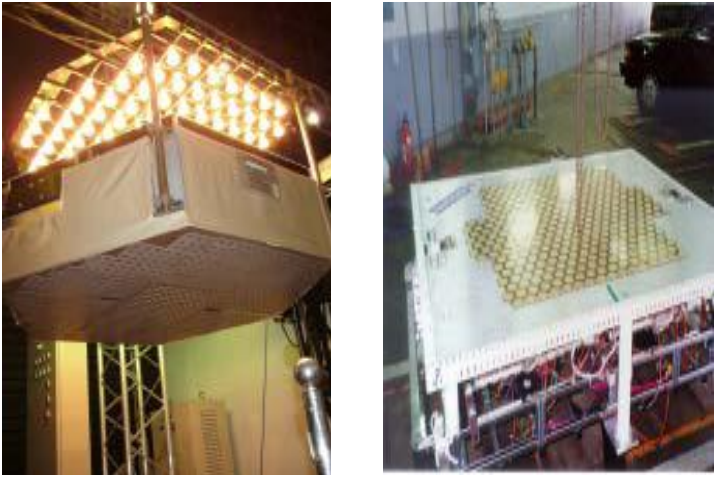

Fig. 11. Phased array used in japanese field mpt experiment.

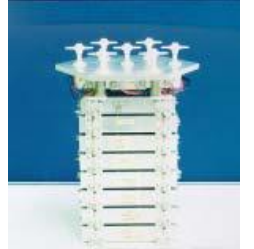

(a)

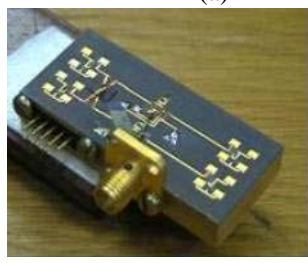

(c)

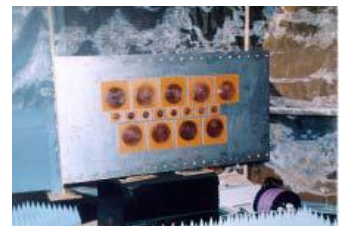

(b)

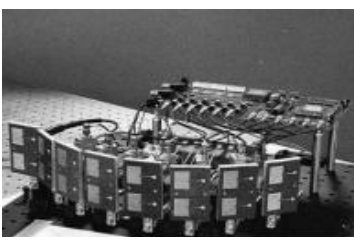

(d)
Fig. 12. Various retrodirective array with phaseconjugate circuits Developed in (a) KyotoUniversity and kobe university in 1987 (2.45GHz), (b) Kyoto university in 1996 (2.45GHz),(c) queen's university $(62-66 \mathrm{GHz})$, (d) Jet propulsion laboratory and university of michigan in $2001(5.9 \mathrm{GHz})$

\section{FUTURE ASPECTS}

\section{A. Power-Generating Solar Satellite Inhabitat}

Japan wants to power up three million houses with wireless energy from space. They have serious plans to send a solar-panel-equipped satellite into space that could wirelessly beam a gig watt-strong stream of power down to earth.

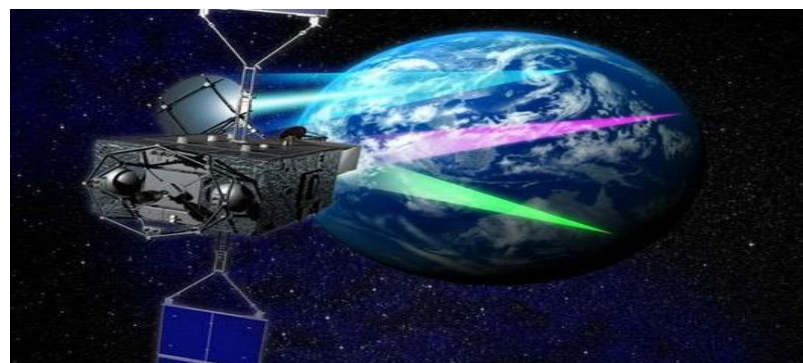

Fig. 13. Japan's wireless, power-generating, solar satellite inhabitat

A small test model is scheduled for launch in 2015. To iron out all the kinks and get a fully functional system set up is estimated to take three decades. A major kink, presumably, is coping with the possible dangers when a 1-gigawatt microwave beam aimed at a small spot on Earth misses its target.

The \$21 billion project just received major backing from Mitsubishi and designer IHI (in addition to research teams from 14 other countries).

\section{B. Third-Generation Wireless Power}

[11].PowerbyProxi has developed a 3G wireless power delivery system. Earlier generations of wireless power technology were based on split transformers consisting of two halves: an input side (primary) and an output side (secondary). Electrical energy applied to the primary is converted to an electromagnetic field that induces a current in the secondary, which passes the energy to a load. The essential difference between earlier generations of wireless power solutions and the one developed by PowerbyProxi is that the PowerbyProxi system offers high efficiency levels in relatively loose coupling arrangements across an air gap or through any nonmetallic substrate.

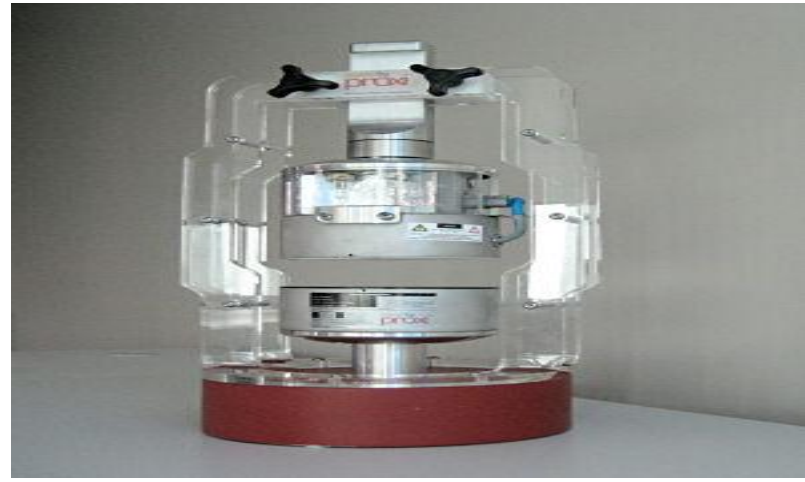

Fig. 14. A demonstration model of the powerbyproxi technology.

PowerbyProxi takes a different approach to wireless power with its patented ProxiWave (Figure) technology. It uses coils to transmit and receive power between a power transmitter 
(PTx) and power receiver (PRx). These coils are analogous to antennas used for radio communication. A converter, powered through AC mains, a truck battery, or other source, is used to drive the PTx coil. The ProxiWave power controller regulates the power flow from the receiving PRx coil to the target electrical device or electronics, depending on their specific power requirements.

\section{MERITS AND DEMERITS}

\section{A. Merits}

The system would reduce the cost of electrical energy used by the consumer and rid the landscape of wires, cables, and transmission towers. The electrical energy can be economically transmitted without wires to any terrestrial distance, so there will be no transmission and distribution loss. The efficiency of the transmission can be as high as 96 or 97 per cent, and there are practically no losses.

\section{B. Demerits}

Calculating the circulating reactive power, it was found that the frequency is very small and such a frequency is very biologically compatible.

\section{IECONOMIC ASPECTS}

In terms of economic theory, many countries will benefit from this service of wireless power transmission. Only private, dispersed receiving stations will be needed. A single resonant energy receiver is required, which may eventually be built into appliances, so no power cord will be necessary. Monthly electric utility bills from old-fashioned, fossil-fuelled, loss prone electrified wire-grid delivery services will be optional.

\section{CONCLUSION}

Wireless power transmission of electrical power can be considered as a large scope in electrical engineering for future prospects of power generation and transfer. Solar power satellites are the future of supplying non conventional energy. The various methods and aspects regarding wireless transmission of electrical power are discussed. The evolution of the technology from the time of Tesla has been overviewed.

\section{ACKNOWLEDGMENT}

Bharati Vidyapeeth Deemed University, Pune. Government Engg College, Thrissur.

\section{REFERENCES}

[1] Colorado Springs notes.

[2] G. E. Maryniak, "Status of international experimentation in wireless power Transmission," Sunset energy counsel, Solar energy, vol. 56, 1996.

[3] R. B. ERB, "International cooperation for the acquisition of space based energy," R Bryan ERB, Solar Energy, vol. 56, 1996.

[4] http://www.nss.org/settlement/ssp/index.html.

[5] P. Vessen, "wireless Power transmission," Leonardo energy; briefing paper.

[6] A. Bomber, "Wireless Power Transmission: An Obscure History, Possibly a Bright Future".

[7] "Wireless energy transfer" Wikimedia Foundation, Inc http://en.wikipedia.org/wiki/Wireless_energy_transfer.

[8] T21 World Global Trends (Accessed March 23, 2006) http://www.t21.ca/energy/index.html.

[9] http://wireless transmission paper/WIRELESS POWER TRANSMISSION__ Yuva Engineers.html.

[10] http://web.mit.edu/newsoffice/2007/wireless-0607.html.

[11] F. Mishriki, "Power Solutions A New Approach to Wireless Power Transfer January 1, 2010," Sensors

[12] Wireless writicity new post, USA.

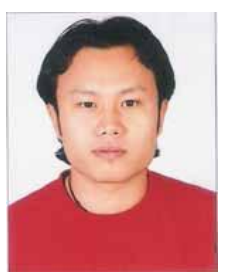

Sagolsem Kripachariya Singh was born in Imphal, Manipur, India on $28^{\text {th }}$ January 1988 . He completed B-tech in Electrical and Electronics Engineering from Govt. Engineering College Thrissur, Kerala in the year 2009. He is presently doing M-tech in Power System at Bharati Vidyapeeth College of Engineering, Pune, Maharastra. 